\title{
How do auditory verbal hallucinations in patients differ from those in non-patients?
}

\author{
Frank Larøi * \\ Department of Psychology: Cognition and Behaviour, University of Liège, Liège, Belgium
}

\section{Edited by:}

Kenneth Hugdahl, University of

Bergen, Norway

Reviewed by:

Kenneth Hugdahl, University of Bergen, Norway

Else-Marie Løberg, University of Bergen, Norway

\section{*Correspondence:}

Frank Larøi, Department of

Psychology: Cognition and

Behaviour, University of Liège, B33,

Sart-Tilman, 4000 Liège, Belgium.

e-mail: flaroi@ulg.ac.be
Auditory verbal hallucinations (AVHs) are experienced by individuals with various clinical diagnoses, such as psychosis, but also a significant minority of healthy individuals from the general population may experience them. Although much research has been carried out the past few decades, the mechanisms and factors underlying the emergence of $\mathrm{AVHs}$ is still poorly understood. One way of clarifying this issue involves comparing AVHs in patient and non-patient populations. In particular, differences between these groups will provide important information concerning the emergence of AVHs. After a general presentation and discussion of the notion of a continuum hypothesis, studies comparing patients with non-patients experiencing AVHs will be reviewed. This will comprise studies examining the phenomenological characteristics of $\mathrm{AVHs}$ in addition to neuroimaging and cognitive studies. Although we are beginning to elucidate important differences on a phenomenological level between these two types of AVHs, far too few studies have directly compared patient and non-patient AVHs in terms of underlying cerebral correlates and cognitive mechanisms. Nevertheless, and based on recent research on phenomenological differences, two issues stand out that need to be addressed, namely, the highly negative emotional content of AVHs in patients and the early onset of AVHs in non-patients populations. Suggestions for future research will be discussed.

Keywords: hallucinations, auditory verbal hallucinations, voice-hearing, continuum, dimension, phenomenology, cognition, neuroimaging

\section{INTRODUCTION}

Hallucinations, or perceptions in the absence of stimuli, may occur in any sensory modality such as auditory, visual, olfactory, gustatory, or tactile. They are very common in individuals with schizophrenia, but may also occur in those suffering from various other psychopathological (e.g., mood disorders, Post-traumatic Stress Disorder, Substance abuse, Borderline Personality Disorder) or neurological (e.g., Dementia, Parkinson's disease, epilepsy) disorders. Studies have furthermore shown that a significant minority of otherwise healthy persons may also experience hallucinations (for reviews of this literature, see Aleman and Larøi, 2008 and Beavan et al., 2011).

Auditory hallucinations represent a particularly rich and varied phenomenology. They involve the perception of a large array of sounds, which, when involving voices, are referred to as auditory verbal hallucinations (AVHs). AVHs are the main focus of the present article as this type of hallucination has been the most often examined in the literature in general, but also in studies comparing hallucinations in clinical groups compared to those experienced by non-clinical or healthy persons.

Despite a significant increase in studies investigating $\mathrm{AVHs}$ in the past few decades (cf. Aleman and Larøi, 2008), they remain poorly understood, in particular regarding underlying mechanisms and factors involved in the development or emergence of AVHs. There exist a number of approaches that can be adopted in order to better elucidate this process. One particularly fruitful recent approach to resolving this issue has been the use of combined epidemiological and longitudinal studies (i.e., following up a representative group of individuals from the general population over time). This line of research can, for example, investigate the nature of the transition from a nonclinical hallucination to a clinical hallucination (i.e., the latter referring to hallucinations in individuals who are in need of professional help for these experiences) and consequently say something about who needs treatment for their AVHs, and why. Two main psychological mechanisms seem to be involved in this. First, response to abnormal experience, such as AVHs, seems to be cognitively mediated by beliefs or appraisals (Garety et al., 2001). That is, the mere experience of AVHs itself might not lead to full-blown psychotic hallucinations, but, for instance, attributing the AVHs to an external malevolent source and giving it personal significance does. Another important determinant of the transition to clinical states may be the level of functional coping that the person mobilizes in the face of stressful experiences such as AVHs. Active coping strategy such as using problem-solving, seeking help, and distraction, seems to generate control over the experiences. In contrast, more passive coping strategies such as going along with and indulging in the content of hallucinations, isolating oneself, getting involved in non-specific activities, do not generate more control over the experiences.

Another way of clarifying the developmental trajectory of AVHs involves the detailed assessment of changes in mental experiences that occur before AVHs develop, especially during early 
(i.e., prodromal) phases of psychosis. A number of changes can be observed that involve a general perceived change in the stream of thoughts of the person (e.g., thoughts no longer shift smoothly and effortlessly from one moment to the next). Individuals may also report that thoughts feel anonymous or spatialized, may acquire a quasi-sensorial concreteness or are experienced as deprived of the tag of "mineness" and familiarity (cf. Larøi et al., 2010; Raballo and Larøi, 2011).

\section{THE CONTINUUM HYPOTHESIS}

Another fruitful approach to help elucidate the emergence of AVHs involves comparing AVHs present in persons with psychosis compared with those AVHs experienced by non-clinical persons or non-patients. As with the combined general population and longitudinal research described above, this line of research provides ways of better differentiating between problematic AVHs that call for treatment (e.g., that are associated with a psychosis diagnosis), and those that do not.

Studies show that some $10-15 \%$ of the healthy population at times experience AVHs (Sommer et al., 2010). In a recent review of the literature (Beavan et al., 2011), which included 17 studies that have examined the prevalence of AVHs in the adult general population, the authors report prevalence rates ranging from 0.6 to $84 \%$. The great majority of the studies included in this review came from Western countries (European countries, the USA) with the exception of two studies from New Zealand and the Philippines. This high degree of variation in reports of AVHs reflects differences in studies in terms of methodology and design (definitions of hallucinations, how items are formulated, the context of the study, participants' socio-demographic characteristics, etc.). For instance, high levels of AVHs prevalence are related to definitions being too broad (e.g., "hearing vocal sounds" or "hearing a voice saying a few words"), periods being too unspecific (e.g., "have you ever experienced ..."), or the inclusion of very common experiences (e.g., "heard own name in a shop"). Moreover, in the few studies that investigated differences in the frequency of hallucinatory experiences among different ethnic groups, it was found that some ethnic groups are more likely than others to report hallucinatory experiences. For example, higher rates were reported for Brazilian, Russian, Caribbean, Hispanic, and Black respondents compared to Western, Caucasian individuals. Other factors were also identified that play a role in the variation of rates such as gender (higher in women) and loss (e.g., decease of a loved one) and trauma (e.g., bullying, sexual abuse).

Nevertheless, these types of findings suggest that hallucinations in general, and AVHs in particular, may be considered as dimensional phenomena lying on continua with normal experiences (Johns and van Os, 2001), and that there are no clear qualitative differences but rather quantitative differences between normality and pathology. It is perhaps preferable to refer to this as a continuum "hypothesis" or "view" (and not a model or theory) as there is still debate concerning the true nature of this continuum, and therefore, hopefully this debate will continue in years to come (David, 2011) as an uncritical acceptance of the continuum hypothesis is clearly to be avoided (Badcock and Hugdahl, 2012).
In this context, it is also important to note that one can distinguish between at least two types of continua (Bentall, 2003; David, 2011):

(1) A continuum of experience, that is within an individual, and which suggests that different kinds of experience (e.g., vivid daydreams, intrusive, and vivid thoughts) may be related to AVHs. This continuum may vary, for instance, from normal thoughts, to intrusive and vivid thoughts, to thoughts resembling voices, and finally to AVHs.

(2) A continuum of risk, which is across several individuals, and indicates that people differ in their proneness to have AVHs experiences, and similarly also in their level of risk to develop problematic or "clinical" AVHs. This continuum may vary, for instance, in terms of frequency-from those with no hallucination-proneness (e.g., that have never had an $\mathrm{AVH}$ or AVH-like experience in their lives), to those who once or sometimes have had such an experience, to those who have it quite frequently, and finally to those who have it very frequently. Similarly, persons on this continuum vary in terms of risk: from those with no risk to develop clinical AVHs at one extreme, to those with a very high risk to do so at the other end.

Thanks to a significant body of studies that has appeared the past few years, it is now possible to attempt to characterize those persons (i.e., those $10-15 \%$ from the general population) who lie on this continuum of risk. At the one end of the continuum are those healthy individuals who experience AVHs very rarely, perhaps often under specific conditions (such as after sleep deprivation or during severe stress), and where AVHs are not very similar to those experienced by patients. Moving toward the other end of the continuum one may plot those individuals who have experienced AVHs from an early age, experience them relatively frequently, that are quite similar to those observed in patients with psychosis, are accompanied by subclinical levels of other symptoms (e.g., paranoid delusions, paranormal beliefs, formal thought disorder, depression, anxiety), and where there may also be a family history of psychiatric illness.

What these individuals all have in common with each other is that the experience of AVHs does not disturb their everyday life functioning - nor does the fact of having AVHs prompt them to seek professional help for these experiences. There is also a relatively good level of insight regarding these experiences and they do not fulfil criteria for a psychiatric or neurological disorder (nor have they ever had one in the past). However, at least two subgroups of non-patients along this continuum may be identified $^{1}$. In one group, or non-patient type i, AVHs are not frequent and are not very similar to AVHs in patients. On the other hand, there are those, i.e., non-patient type ii, who experience AVHs very frequently and these are very similar to those experienced in patients. These two groups, moreover, probably plot somewhat differently on the above-mentioned continua. Regarding the continuum of experience, non-patients i plot toward the left, whereas

${ }^{1}$ Please note that there is most definitely overlap between these two subgroups, so they cannot be viewed as clearly distinct groups. 
non-patients ii plot very close to the right. Similarly, turning to the continuum of risk, one may suggest that non-patients i plot to the left, whereas non-patients ii are situated to the right of the continuum. Also, this distinction will be important in the present article as studies in the literature have included nonpatient groups of either type i or type ii. Important to underline here is that individuals of type i non-patients may include those based on vague and broad criteria mentioned earlier, and therefore AVHs experienced in this group of individuals are relatively far-removed from those experienced by patients. Moreover, as type ii non-patients experience AVHs that are closest to those experienced by psychotic patient groups, studies including these non-patient individuals will be emphasized as much as possible.

\section{INCLUSION OF PATIENT VS. NON-PATIENT AVHS IN STUDIES}

Studies that include non-patient groups in the context of AVHs have either (1) only included non-clinical participants or (2) have compared non-clinical and clinical participants in a direct manner, that is, in the same study with the same methodology. Both approaches have their merits, although it will be argued that the latter study design is the preferred of the two.

Studies including only non-clinical participants are interesting for a number of reasons. This study design allows one to avoid confounding effects (e.g., on cognitive test performance and neuroimaging data) associated with long-term medication and institutionalization, illness duration, low level of education, etc., present in patient populations. Also, studies that only include non-clinical persons provide an examination (and eventual validation) of the various components of the continuum hypothesis. These components may include, for example, distributional, phenomenological, developmental, and etiological components (Aleman and Larøi, 2008). The distributional component refers to the fact that AVHs should be present not only in subjects identified as "clinical cases" but also in a proportion of subjects from the general population that does not fulfil the clinical criteria of a patient. The phenomenological component consists of revealing a sufficient degree of inter-group similarity, in addition to a large degree of within-group variation, in terms of phenomenological characteristics of AVHs (e.g., degree of control, frequency, duration, emotional response, and content). The developmental component relates to aspects associated with the genesis of AVHs and that they should also be continuous between pathological and non-pathological samples. That is, factors identified as important demographic risk factors in clinical cases of hallucinations (e.g., younger age, higher level of urbanicity, lower income, lower level of education, unemployment, single marital status) should also be associated with the presence of hallucinations in non-clinical subjects. Finally, the etiological component maintains that clinical and non-clinical populations should share common ground in terms of underlying etiological mechanisms (e.g., cognitive, psychological, and neural mechanisms) of AVHs.

In contrast, comparing non-patient with patient AVHs in the same study has the vital advantage of being able to directly compare, as it were, these two "types" of AVHs. Moreover, and most importantly, such a study design is able to tease out both similarities and differences between these two groups. Where similarities are found, this points to mechanisms and factors that are not involved in the emergence of problematic AVHs. In contrast, where differences are found between these two groups, this indicates both possible factors and mechanisms involved in developing problematic AVHs, but also so-called "protective" mechanisms and factors that help prevent someone from developing problematic AVHs. Thus, including clinical and non-clinical individuals experiencing AVHs helps clarify which features of AVHs do not do necessarily reflect pathology (e.g., in cases where there are similarities between clinical and non-clinical groups) and, on the other hand, those features that may reflect pathology (e.g., in cases where the feature is present in clinical groups, but not in non-clinical groups). Such an approach clearly has clinical implications and will help identify new types of techniques to add to the ever growing arsenal of intervention strategies available for treating and managing hallucinations (cf. Larøi and Aleman, 2010).

What follows is a review of those studies that have done just that-compared clinical and non-clinical AVHs in the same study. Also, and for the reasons mentioned earlier, those studies including non-patients of type ii will be given priority. Moreover, these two types of AVHs will be compared in terms of their phenomenology, cerebral correlates, and cognitive mechanisms. Finally, emphasis will be made regarding eventual differences between patient and non-patient AVHs and thus another important characteristic of studies is that the study design allows for a clear demarcation of similarities and differences between groups.

\section{PHENOMENOLOGICAL STUDIES}

The term "phenomenology" may be used in a number of different ways. However, in this context it signifies the detailed description of the clinical and/or descriptive features of signs and symptoms observed in psychopathological conditions. In the case of AVHs, this refers to evaluating such characteristics as frequency, controllability, content, personification, emotional valence, duration, localization, loudness, and number of voices.

\section{INCLUSION OF A NON-PATIENT GROUP}

Although the phenomenological characteristics of AVHs in schizophrenia have been relatively well-described and studied in the scientific literature, this is clearly not the case with AVHs in non-patient populations. One exception is a recent and welldesigned study by Sommer et al. (2010), whereby a group of non-clinical individuals experiencing AVHs is compared with a group of matched healthy subjects. One of the particular strengths of this study, and its study design, is the manner in which nonclinical individuals are carefully recruited, and this according to both strict and clear-cut criteria. A website providing information about hearing voices was created with various questions, including two items from the Launay-Slade Hallucinations Scale (LSHS, Larøi et al., 2004: "In the past I have had the experience of hearing a person's voice and then found that there was noone there," "I have been troubled by hearing voices in my head"). Those with high scores on these two items were selected and were interviewed by telephone to confirm that they met the following criteria: (1) voices were distinct from thoughts and had a "hearing" quality, (2) voices were experienced at least once a month, (3) no diagnosis or treatment for psychiatric disorders other than 
depressive or anxiety disorders in remission, (4) no alcohol or drug abuse for at least three months, (5) no chronic somatic disorder, (6) 18 years of age or older, and (7) four Dutch-born grandparents (to restrict heterogeneity for later genetic studies). This resulted in 103 individuals with AVHs and 60 control participants. Control participants scored 0 on both LSHS items, met the above-mentioned criteria, and were matched for sex, age, and education.

Characteristics of hallucinations were assessed with the Psychotic Symptom Rating Scales (PSYRATS; Haddock et al., 1999) and the LSHS. Results for the AVH group showed that the mean number of years experiencing AVHs was 29 years, and the mean age at first experiencing voices was 14 years. The types of voices experienced included commenting voices $(18 \%)$ and voices speaking with each other (11\%). The majority (71\%) never heard AVHs with negative content only, 25\% experienced both positive and negative AVHs, and 4\% experienced AVHs with a negative content only. A great number (91\%) reported no disturbance of daily life by their AVHs. AVHs were attributed to an external source in $58 \%$ of participants, mostly benevolent spirits. Based on structured clinical interviews, they did not have clinically relevant delusions, disorganization or negative or catatonic symptoms and did not meet criteria for cluster A personality disorder. Global level of functioning (Global Assessment of Functioning) was found to be lower than in the controls (82 and 87 , respectively), although scores were within the normal range and lower levels was predominately attributable to lower levels of occupational functioning in the AVH group. There were significantly higher scores on the Schizotypal Personality Questionnaire (for both Total score and for scores on the Cognitive-perceptual, Disorganzational, and Interpersonal sub factors) and the Peters et al. Delusion Inventory (PDI), indicating a general increased schizotypal and delusional tendency in the AVH group. History of childhood trauma and family history of axis I disorders were also significantly more prevalent in these individuals compared to controls.

In summary, the AVH group experienced AVHs quite frequently and from an early age, but did not fulfil criteria for any clinical disorders. The AVHs they experience are both similar to those in (psychotic) patient groups (e.g., commenting voices, voices speaking with each other) but at the same time do not resemble those experienced by patients in that they very rarely have a negative content. The two groups did not differ considerably in terms of global social functioning, but did differ in terms of the presence of subclinical symptoms, history of childhood trauma, and family history of axis I disorders. According to the authors, these findings suggest that AVHs in otherwise healthy individuals is not an isolated phenomenon but, rather, part of a general vulnerability for schizophrenia. This might furthermore suggest a genetic predisposition for schizophrenia in the AVH group (e.g., based on a family history of axis I disorders), which, however, is also in interaction with various environmental risk factors (e.g., childhood trauma).

\section{INCLUSION OF NON-PATIENT AND PATIENT GROUPS}

As already mentioned, whilst studies only including non-patients participants, such as the above-mentioned study, provide us with important information concerning AVHs, the ideal study design is to compare AVHs in patient and non-patient populations. At present, only three studies (Leudar et al., 1997; Honig et al., 1999; Daalman et al., 2011) have compared AVHs in non-patients with patients suffering from psychosis.

The most recent study is Daalman et al. (2011). In this study, non-patient individuals were recruited in the same manner as described in Sommer et al. (2010). The non-patients $(n=111)$ did not meet criteria for DSM-IV diagnosis, whilst the patient group ( $n=118$ ) consisted of outpatients with a confirmed psychotic disorder. Both groups experienced AVHs at least once a month for over one year. The measure of AVHs included the PSYRATS (Haddock et al., 1999), in addition to five supplementary questions assessing aspects not included in the PSYRATS (e.g., age at onset, number of voices, personification, explanation of origin).

Results revealed higher scores for patients for the following AVH-related items of the PSYRATS: more negative content, higher distress and disruption of daily life, greater frequency, longer duration, and less controllability. Other phenomenological characteristics, such as perceived location of voices (heard inside or outside the head), loudness, number of voices, and personification (attribution to a real and familiar person), did not differ between the two groups. Interestingly, a very early mean onset of AVHs was observed in non-patients (12 years of age) and this was much lower than in patients (21 years). The beliefs that individuals held about the origin of hallucinations also differed. Non-patients frequently attributed their voices to spiritual sources (spirits of deceased people, guardian angels, entities, angels, presences), whereas patients often attributed their voices to real people, such as the secret police, telepathic people, drug gangs, or malevolent neighbors.

Previous studies of this kind have reported similar results. For instance, Honig et al. (1999) compared non-patients with two clinical groups (schizophrenia, dissociative disorders), all of whom were experiencing AVHs. The non-patient group was recruited via the local media, and included individuals with no previous psychiatric history, no recent onset of another psychiatric disorder and a score on the Dissociative Experiences Scale (Bernstein and Putnam, 1986) below 30. Hallucination assessment was carried out by means of a semi-structured interview with open-ended and closed questions relating to characteristics of the hallucinations (e.g., frequency, number, form, content, emotional qualities, gender, and age), history of the voices, circumstances related to the onset of hearing voices, present triggers, personal interpretation of the voices, coping strategies, and life history including traumatic experiences. Results revealed that non-patients generally felt in control of the experience, in contrast to the two patient groups. All three groups reported positive voices, but there were group differences in negative voices: these were reported by all in the schizophrenia group, 93\% in the dissociative group, but only $53 \%$ in the non-patient group, suggesting that negative voices differentiated the groups. Moreover, the two patient groups were afraid of their voices, and reported significant disturbances in their daily life, and that the voices were critical and troublesome. A larger percentage of the non-clinical participants had an age of onset before 12 years of age (40\%) compared 
to the dissociative (33\%) and schizophrenia (11\%) groups. In terms of frequency, the daily and continuous experiences of voices were more frequent in the patient groups than in the non-patient group. Loci (AVHs experienced inside vs. outside the person) were similar in all three groups.

Finally, based on structured interviews, Leudar et al. (1997) examined specific pragmatic properties of AVHs (e.g., number of voices, characteristics that individuate the voices, sequential characteristics of the dialogs between voice hearers and their voices, dialogical positioning of voices hearers, voices and other individuals, and how the voices influence voice hearers' activities) and found no major differences in the structure and function of the AVHs of these two groups (non-patients and patients with schizophrenia both experiencing AVHs). However, they did note that patients with schizophrenia were more likely to identify their voices as being public figures, and that their voices were more likely to instigate violence. The voices of the non-patients were more likely to evaluate others, to have mundane content, and to be identified as being the voices of family members.

Thus, on a phenomenological level, there appear to be both similarities and differences between AVHs in patients and nonpatients. Concerning similarities, this seems to be related to more perceptual and/or acoustic aspects such as such as localization, loudness, number of voices, and personification. Differences seem to be related to reactions to the voices and their effect on everyday functioning, in particular the negative emotional content of AVHs and the distress they elicit, in addition to higher frequency and less control. One other important difference is the age of onset of voices-around 12 years in non-patients compared to 21 in patients. As mentioned earlier, it is important to take into account, and better understand, these differences as they provide us with important clues as to why certain persons with these experiences are able to function normally in everyday life, whilst others (i.e., patients) are not.

\section{NEUROIMAGING STUDIES}

There has been an abundance of neuroimaging studies of AVHs in schizophrenia the past few years (cf. reviews and meta-analyses by Allen et al., 2008; Jardri et al., 2011; Kompus et al., 2011). In general, a distributed brain network has been shown to be implicated in the experience of AVHs. In their review of the literature, Allen et al. (2008) point to the involvement of secondary (and sometimes primary) sensory cortices, prefrontal, subcortical, and cerebellar regions. Recently, Jardri et al. (2011) performed a meta-analysis of studies $(n=10)$ examining cortical activation (fMRI, PET) during AVHs and found that they were associated with increased activity in fronto-temporal (especially those involved in speech generation and perception) and medial temporal (hippocampal/parahippocampal regions) areas. In a highly innovative recent study, Kompus et al. (2011) performed metaanalyses of neuroimaging studies (fMRI, PET) examining patients with schizophrenia (compared to healthy controls) during the processing of auditory stimuli (11 studies), and studies including patients experiencing AVHs in the absence of auditory stimuli (12 studies). The results revealed increased activation in the left primary auditory cortex and the right rostral prefrontal cortex when experiencing AVHs (and in the absence of an external stimulus) but, paradoxically, activation in these areas decreased in the presence of auditory stimulation in patients when compared with healthy controls. The authors suggest that this "paradox" is either caused by an attentional bias toward internally generated information and/or the failure of a default network to deactivate when auditory processing areas are engaged by external stimuli.

Although much research has been devoted to examining cerebral correlates in patients experiencing AVHs, only one study has compared patients and non-patients with AVHs (Diederen et al., in press). In this study, 21 non-psychotic subjects with AVHs and 21 matched patients with psychosis were asked to indicate the presence of AVHs during functional magnetic resonance imaging (fMRI). The non-patients were recruited in the same manner as described in Sommer et al. (2010). The patients with psychosis matched the non-patients for both demographic factors (e.g., age, sex, handedness) and for the total duration of AVHs, the mean duration of AVHs and the number of AVHs experienced during fMRI scans. Furthermore, for all participants, the AVHs had to be present with a frequency of at least four AVH episodes per scan, had to last at least $50 \mathrm{~s}$, and participants had to clearly indicate both AVH onset and offset.

The results revealed that several areas were significantly activated during AVHs in both groups including the bilateral inferior frontal gyri, insula, superior temporal gyri, supramarginal gyri and postcentral gyri, left precental gyrus, inferior parietal lobule, superior temporal pole and right cerebellum. The activation of these areas during AVHs is in line with previous research including patients with psychosis with AVHs. Furthermore, and importantly, no significant differences in activation during AVHs between the groups were found. These findings suggest the involvement of the same brain areas in non-patient and patient groups during AVHs.

\section{COGNITIVE STUDIES}

There has also been much research examining the cognitive mechanisms involved in AVHs (cf. Aleman and Larøi, 2008). In general, there seems to be a consensus that AVHs occur when a private event is misattributed to a source that is external or alien to the self. A two-step process is most probably implicated in this (Larøi and Woodward, 2007) involving: (1) a form of autonoetic agnosia, or an inability to identify self-generated mental events and then (2) a misattribution, whereby these self-generated mental events are misattributed as coming from another (e.g., a non-self, external, alien) source. A number of cognitive mechanisms that might underlie this process have been identified in the literature and found to be associated with AVHs in patients with psychosis. These include inhibition, source memory, contextual memory, verbal self-monitoring, and metacognitive beliefs. A review of even a fragment of these studies is impossible in the context of this article and, therefore, the interested reader may refer to the many excellent reviews that exist on the topic (Seal et al., 2004; Ditman and Kuperberg, 2005; Nieznański, 2005; Aleman and Larøi, 2008).

What interests us most here, though, is whether these proposed cognitive mechanisms are also present in non-patient groups experiencing AVHs. However, although many studies have examined the role of these cognitive mechanisms in both patient 
and non-patient groups, no study has done so in the same study ${ }^{2}$. Furthermore, only some form of non-patient type i have been included in these studies, unfortunately, leaving no study in the literature examining the role of cognitive mechanisms in type ii non-patients.

As an illustration, one proposed mechanisms involved in AVHs, verbal self-monitoring, will be described in general and in the context of studies of non-patients. One prominent model (Frith, 1987, 1992) maintains that AVHs are the result of defective self-monitoring, whereby defective monitoring of verbal thoughts leads to a failure in the recognition of one's own thoughts as self-generated and, as a consequence, these thoughts are misidentified as externally generated voices. A series of studies has tested this hypothesis using a verbal self-monitoring task. This task involves asking participants to pronounce a word, and then providing immediate auditory verbal feedback to participants via earphones. This verbal feedback may be: (1) the participant's own voice fed back to the participant, (2) another person's voice (albeit of the same gender) fed back to the participant, (3) the participant's own voice fed back to the participant but where the voice is distorted, or (4) another person's voice (of the same gender) fed back to the participant but where the voice is distorted. Participants are then asked to say if the verbal feedback is their own voice, someone else's voice or not sure. In general, results have found that patients with schizophrenia, and in particular those with AVHs, make more errors than controls, and moreover with a particular bias toward misattributing their own distorted voice to another person. Important to note is that the evidence that AVHs arise through a deficit in verbal self-monitoring alone is equivocal (Allen et al., 2007) as, for instance, this finding has not been found to be necessarily specific in patients with AVHs and many times is also associated with other symptoms, such as delusions.

Nevertheless, this line of research provides us with a good example, as this same task has also been tested in various forms of non-patient groups. In Johns et al. (2010), persons with At Risk Mental State (ARMS) were compared with a group of healthy controls on this task. The former group consisted of individuals who experience attenuated forms of psychotic symptoms—symptoms that are similar to full psychotic symptoms but less severe and associated with greater insight. Results revealed impaired verbal self-monitoring in the ARMS group compared to controls (i.e., greater number of errors when own speech was distorted and more likely to misattribute distorted own speech to another source). Furthermore, the authors mention that the verbal selfmonitoring deficit seemed "to be less marked than in patients with schizophrenia" although this could not be directly tested due to the fact that a group of psychotic patients was not included in the study. Allen et al. (2006) used a similar task, albeit in a group of healthy individuals (University undergraduates) who completed scales measuring proneness toward hallucinations (LSHS) and delusions (PDI, Peters et al., 1999). Results showed that misattribution errors on the verbal self-monitoring task for own

\footnotetext{
${ }^{2}$ Kindly note that Badcock and Hugdahl (2012) have provided an excellent review and discussion of possible patterns of shared and distinct cognitive (and neural) AVH mechanisms in clinical and non-clinical populations.
}

distorted speech was significantly correlated to scores on the PDI but that there was only a trend for a correlation with scores on the LSHS. Finally, Versmissen et al. (2007) compared patients with psychosis, and subjects from the general population with a high level of psychotic experiences, on the verbal self-monitoring task. The authors did not report significant differences between the groups and, furthermore, mention that there were no abnormal performances on the task.

Thus, in terms of cognitive studies-even amidst the multitude of possible cognitive mechanisms implicated in AVHs based on research with psychotic patients, studies have tested these mechanisms in non-patient groups alone-but no study to date has included both patient and non-patient groups. Furthermore, at present, no study has included the all-important non-patient type ii group. This results in it being highly difficult to suggest which cognitive mechanisms are present in non-patient $\mathrm{AVHs}$, and impossible to say anything about which mechanisms are involved (or not) in non-patient compared to patient AVHs. Verbal self-monitoring studies were used to illustrate this.

\section{CONCLUSIONS}

In general, some studies have managed to tease out both similarities and differences in patient and non-patient AVHs. The greatest progress, however, is probably on a phenomenological level. Here, it seems that differences between patients and nonpatients regards the reactions to AVHs and the effect AVHs have on everyday life functioning. In contrast, in terms of cerebral areas involved in patient vs. non-patient AVHs, there are far too few studies examining this issue. The only existing study of this kind did not observe any significant differences between cerebral activation in patient and non-patient AVHs. This finding is interesting in itself, but evidently needs to be replicated in future studies. Regarding cognitive mechanisms, unfortunately, there are no studies that have directly compared clinical and non-clinical AVHs and, therefore, it is difficult to determine whether or not cognitive mechanisms (inhibition, source memory, verbal selfmonitoring, meta-cognitive beliefs) found to be present in patient groups also play a role in AVHs in non-patient groups. Studies that have included non-clinical participants (type ii) suggest that the same cognitive mechanisms found to be involved in AVHs in patients are also involved in non-patients. Whether they are to the same extent as in patient groups, or whether deficit levels are observed in these non-patient AVHs, however, is not known and needs to be examined in future studies.

On a more general note, and as mentioned by Badcock and Hugdahl (2012), there is a grave need for future studies to more directly examine similarities and differences of patient and non-patient AVHs on phenomenological, cognitive, cerebral (and other) levels by including both types of individuals. It was mentioned that those non-patient studies examining, for instance, cognitive mechanisms suggest that these same mechanisms are implicated in both patient and non-patient AVHs. However, as this has yet to be directly examined in studies, this can only be characterized as being a tentative interpretation. Indeed, it is possible that certain cognitive mechanisms are only implicated in patient AVHs, yet to date studies have not been designed to test this important hypothesis in a direct manner. We have 
perhaps (unknowingly) been too intent on identifying similarities between patient and non-patient AVHs and at the same time have not fully realized the significance of detecting differences between these two. Consequently, future studies will also need to ensure that study design will allow a clear demarcation of similarities and differences between groups. Finally, the approach adopted by Sommer and colleagues regarding recruitment of non-patient individuals with AVHs is the best method as it allows for the identification of non-patients of type ii, which are very close on a number of levels to those AVHs experienced by patients with psychosis. Hopefully other researchers will adopt a similar recruitment method in forthcoming studies.

As a result of recent phenomenological studies, and in particular Daalman et al. (2011), two major differences seem to stand out when comparing patient and non-patient AVHs, and therefore, merit further discussion: (1) the negative emotional content of AVHs in patients and the distress they elicit, the higher frequency, and less controllability, and (2) the lower age of onset of AVHs in non-patients (12 years) compared to patients (21 years). Negative content might explain both higher levels of distress and frequency, and less control. That is, experiencing AVHs with a highly negative content will render these experiences distressful for individuals, leading to increased frequency and consequently to less controllability due to a number of processes such as a rebound effect. What remains to be explained is why patients' AHVs are so negative. Studies have shown that childhood trauma is present in non-patients experiencing AVHs (cf. Sommer et al., 2010) and a growing body of research has similarly revealed that patients with psychosis with AVHs have experienced early trauma (cf. Fowler et al., 2006) ) $^{3}$ Moreover, certain authors have convincingly argued that AVHs should be considered as a dissociative experience and, in particular, as an after-effect of traumatic or highly stressful experiences (cf. Moskowitz and Corstens, 2007). This might also contribute to an understanding as to why nonpatient individuals who experience AVHs have such an early onset (12 years of age), that is, in a period where such traumatic experiences may have occurred. Escher et al. (2004) report that in their cohort of 80 children and adolescents who experienced AVHs, in about $75 \%$ of them, the onset of AVHs was related to traumatic events or circumstances beyond their control (e.g., death of someone close, problems in the home situation or school, sexual abuse, long-term physical illness, etc.). Another key to trying to understand these findings may be related to emotion regulation strategies. A study (van der Meer et al., 2009) using the Emotion Regulation Questionnaire (ERQ; Gross and John, 2003) has shown that patients with psychosis, compared to healthy controls, tend to use suppression more often and reappraisal strategies less often ${ }^{4}$. This pattern in relation to symptomatology was not examined in this study, however, a recent study (Badcock et al., 2011), also using the ERQ, found that, within their group of patients with schizophrenia, an increased use of

\footnotetext{
${ }^{3}$ As this has not yet been done, it would be interesting for future studies to directly compare patient and non-patient groups experiencing AVHs in terms of early traumatic experiences (e.g., timing, frequency, type of events, etc.).

${ }^{4}$ Kindly note, however, that some studies have not replicated this finding (cf. Henry et al., 2007, 2008).
}

suppression correlated positively with AVH severity (frequency, duration, loudness). Thus, non-patients, in face of traumatic and/or highly stressful situations develop AVHs as a dissociative reaction to these events but thanks to the increased use of adaptive (e.g., reappraisal) and the decreased use of maladaptive emotion regulation strategies (e.g., suppression), these individuals are able to adequately cope with the emotional force of these experiences and, therefore, they will not have a major influence on the content of the AVHs in these persons. In contrast, it may be proposed that patients with psychosis who experience AVHs who are also confronted with highly stressful or traumatic events resulting in dissociative experiences such as AVHs, and that due to the use of maladaptive emotion regulation strategies (and the absence or less frequent use of more adaptive strategies) - these individuals are not able to appropriately cope with the emotional intensity of these experiences resulting in these experiences having a profound influence on the content of their AVHs. Indeed, the use of maladaptive emotion regulation strategies, such as suppression, is related to a number of non-beneficial consequences such as an inability to reduce the experience of unwanted emotions. The precise mechanisms and processes involved in this (i.e., the dissemination of these strong emotions into the contents of AVHs), however, are not known and, therefore, need to be directly examined in future studies. Unfortunately, though, no study has directly examined patients compared to non-patients with AVHs regarding their use of emotional regulation strategies. However, and as mentioned earlier, in order for future studies to maximize possibilities of identifying similarities — but most important differences-between patient and non-patient groups with AVHs (and furthermore due to the complex and multi-dimensional nature of emotional regulation itself), it will probably be best to include an extensive and multi-dimensional battery of emotion regulation measures such as the Cognitive Emotion Regulation Questionnaire (Garnefski et al., 2001) that includes a number of adaptive (e.g., acceptance, positive refocusing, refocus on planning, positive reappraisal, putting into perspective) and maladaptive (e.g., self-blame, rumination, catastrophizing, blaming others) strategies, and not just the ERQ, which evaluates only suppression and reappraisal. Furthermore, in order to properly test this hypothesis, such investigations would have to take place early in the individuals' lives, such as in childhood. Another issue that needs to be addressed is why patients develop their AVHs much later (i.e., late adolescence/early adulthood) and not in childhood when the majority of these traumatic events are likely to occur.

Finally, a number of other issues also need to be examined in future studies. It was mentioned that combined epidemiological and longitudinal studies have shown that the transition from a non-clinical AVH to a clinical AVH is related to appraisals of these experiences and how people cope with them. Concerning the latter point, research presented in the present article suggests that it may also be important to examine how people cope with emotions in general (via emotion regulation strategies) and not just how they cope with the AVHs themselves. Regarding the former point, studies (cf. Birchwood and Chadwick, 1997) show that much emotional (e.g., distress depression, anxiety) and voice-driven behavior (e.g., maladaptive coping strategies) in patients experiencing AVHs is mediated by the beliefs they 
have about the voice's identify (e.g., a malevolent, omnipotent, or omnipresent voice). For instance, voices believed to be malevolent provoke fear and anger and are resisted, whereas benevolent voices are associated with positive affect. It would be interesting to examine the types of beliefs that non-patients have regarding their voices. In this context, it is likely that non-patients do not frequently hold beliefs that their voices are malevolent and omnipotent, as Daalman et al. (2011) showed that non-patients often attribute their voices to (benevolent) spiritual sources (such

\section{REFERENCES}

Aleman, A., and Larøi, F. (2008). Hallucinations: The Science of Idiosyncratic Perception. Washington, DC: American Psychological Association.

Allen, P., Aleman, A., and McGuire, P. K. (2007). Inner speech models of auditory verbal hallucinations: evidence from behavioural and neuroimaging studies. Int. Rev. Psychiatry 19, 409-417.

Allen, P., Freeman, D., Johns, L., and McGuire, P. (2006). Misattribution of self-generated speech in relation to hallucinatory proneness and delusional ideation in healthy volunteers. Schizophr. Res. 84, 281-288.

Allen, P., Larøi, F., McGuire, P. K., and Aleman, A. (2008). The hallucinating brain: a review of structural and functional neuroimaging studies of hallucinations. Neurosci. Biobehav. Rev. 32, 175-191.

Badcock, J. C., and Hugdahl, K. (2012). Cognitive mechanisms of auditory verbal hallucinations in psychotic and non-psychotic groups. Neurosci. Biobehav. Rev. 36, 431-438.

Badcock, J. C., Paulik, G., and Maybery, M. T. (2011). The role of emotion regulation in auditory hallucinations. Psychiatry Res. 185, 303-308.

Beavan, V., Read, J., and Cartwright, C. (2011). The prevalence of voicehearers in the general population: a literature review. J. Ment. Health 20, 281-292.

Bentall, R. P. (2003). Madness Explained: Psychosis and Human Nature. London: Penguin Books.

Bernstein, E. M., and Putnam, F. W. (1986). Development, reliability and validity of a dissociation scale. J. Nerv. Ment. Dis. 174, 727-735.

Birchwood, M., and Chadwick, P. (1997). The omnipotence of voices: testing the validity of a cognitive model. Psychol. Med. 27, 1345-1353.

Daalman, K., Boks, M. P. M., Diederen, K. M. J., de Weijer, A. D., Blom, J. D., Kahn, R., and Sommer, I. E. C. (2011). The same or different? Auditory verbal hallucinations in healthy and psychotic individuals. J. Clin. Psychiatry 72, 320-325.

David, A. S. (2011). Why we need more debate on whether psychotic symptoms lie on a continuum with normality. Psychol. Med. 40, 1935-1942.

Diederen, K. M. J., Daalman, K., de Weijer, A. D., Neggers, S. F. W., van Gastel, W., Blom, J. D., Kahn, R. S., and Sommer, I. E. C. (in press). Auditory hallucinations elicit similar brain activation in psychotic and nonpsychotic individuals. Schizophr. Bull.

Ditman, T., and Kuperberg, G. R. (2005). A source-monitoring account of auditory verbal hallucinations in patients with schizophrenia. Harv. Rev. Psychiatry 13, 280-299. Delespaul, P., van Os, J., Romme, M. (2004). Determinants of outcome in the pathways through care for children hearing voices. Int. J. Soc. Welf. $13,208-222$.

Fowler, D., Freeman, D., Steel, C., Hardy, A., Smith, B., Hackmann, C., Garety, P., Kuipers, E., and Bebbington, P. (2006). "The catastrophic interaction hypothesis: how do stress, trauma, emotion and information processing abnormalities lead to psychosis?" in Trauma and Psychosis, eds W. Larkin and A. P. Morrison (Hove: Routledge), 101-124.

Frith, C. D. (1987). The positive and negative symptoms of schizophrenia reflect impairments in the perception and initiation of action. Psychol. Med. 17, 631-648.

Frith, C. D. (1992). The Cognitive Neuropsychology of Schizophrenia. Hove: Lawrence Erlbaum Associates.

Garety, P. A., Kuipers, E., Fowler, D., Freeman, D., and Bebbington, P. E. (2001). A cognitive model of the positive symptoms of psychosis. Psychol. Med. 31, 189-195.

Garnefski, N., Kraaij, V., and Spinhoven, P. (2001). Negative life events, cognitive emotion regulation, and emotional
Escher, A., Morris, M., Buiks, A.,

as spirits of deceased people, guardian angels, entities, angels, and presences) and furthermore that AVHs in non-patients rarely provoke negative emotions such as distress and are rarely negative in content. Nevertheless, an examination of this issue merits to be carried out. Similarly, it would be noteworthy to examine if changes (described earlier in the article) in mental experiencessuch as changes in the stream of thought-occurring before AVHs develop during prodromal phases in patients, also occur in non-patients.

problems. Pers. Individ. Dif. 30, 1311-1327.

Gross, J. J., and John, O. P. (2003) Individual differences in two emotional regulation processes: implications for affect, relationships and well-being. J. Pers. Soc. Psychol. 85 348-362.

Haddock, G., McCarron, J., Tarrier, N., and Faragher, E. B. (1999). Scale to measure dimensions of hallucinations and delusions: the psychotic symptom rating scales (PSYRATS) Psychol. Med. 29, 879-889.

Henry, J. D., Green, M. J., De Lucia, A., Restuccia, C., McDonald, S. and O'Donnell, (2007). Emotion dysregulation in schizophrenia: reduced amplification of emotional expression is associated with emotional blunting. Schizophr. Res. 95, 197-204.

Henry, J. D., Rendell, P. G., Green, M. J., McDonald, S., and O'Donnell, M. (2008). Emotion regulation in schizophrenia: affective, social and clinical correlates of suppression and reappraisal. J. Abnorm. Psychol. 117, 473-478

Honig, A., Romme, M., Ensink, B. J., Escher, A., Pennings, M., Devries, M. (1999). Auditory hallucinations: a comparison between patients and non-patients. J. Nerv. Ment. Dis. $186,646-651$

Jardri, R., Pouchet, A., Pins, D., and Thomas, P. (2011). Cortical activations during auditory verbal hallucinations in schizophrenia: a coordinate-based meta-analysis. Am. J. Psychiatry. 168, 73-81.

Johns, L. C., Allen, P., Valli, I., WintonBrown, T., Broome, M., Woolley, J., Tabraham, P., Day, F., Howes, O., Wykes, T., and McGuire, P. (2010). Impaired verbal self-monitoring in individuals at high risk of psychosis. Psychol. Med. 40, 1433-1442.

Johns, L. C., and van Os, J. (2001). The continuity of psychotic experiences in the general population. Clin. Psychol. Rev. 21, 1125-1141.

Kompus, K., Westerhausen, R. and Hugdahl, K. (2011). The "paradoxical" engagement of the primary auditory cortex in patients with auditory verbal hallucinations: a meta-analysis of functional neuroimaging studies. Neuropsychologia 49, 3361-3369.

Larøi, F., and Aleman, A. (2010). Hallucinations: A Guide to Treatment and Management. Oxford: Oxford University Press.

Larøi, F., Marczewski, P., and van der Linden, M. (2004). Further evidence of the multi-dimensionality of hallucinatory predisposition: factor structure of a modified version of the Launay-slade hallucinations scale in a normal sample. Eur. Psychiatry 19, 15-20.

Larøi, F., and Woodward, T. S. (2007). Hallucinations from a cognitive perspective. Harv. Rev. Psychiatry 15, 109-117.

Larøi, F., de Haan, S., Jones, S., and Raballo, A. (2010). Auditory verbal hallucinations: dialoguing between the cognitive sciences and phenomenology. Phenomenol. Cogn. Sci. 9, 225-240.

Leudar, I., Thomas, P., McNally, D., and Glinski, A. (1997). What voices can do with words: pragmatics of verbal hallucinations. Psychol. Med. 27, 885-898.

Moskowitz, A., and Corstens, D. (2007). Auditory hallucinations: psychotic symptom or dissociative experience? J. Psychol. Trauma 3, 35-63.

Nieznański, M. (2005). "Reality monitoring failure in schizophrenia: relation to clinical symptoms and impairment of self-concept," in Progress in Schizophrenia Research, ed J. E. Pletson (Hauppauge: Nova Science Publishers), 45-76.

Peters, E. R., Joseph, S. A., and Garety, P. A. (1999). Measurement of delusional ideation in the normal population: introducing the PDI (Peters et al. Delusions Inventory). Schizophr. Bull. 25, 553-576.

Raballo, A., and Larøi, F. (2011). Murmurs of thought: phenomenology of hallucinatory consciousness in impending psychosis. Psychosis 3 , 163-166. 
Seal, M. L., Aleman, A., and McGuire, P. (2004). Compelling imagery, unanticipated speech and deceptive memory: neurocognitive models of auditory verbal hallucinations in schizophrenia. Cognit. Neuropsychiatry 9, 43-72.

Sommer, I. E., Daalman, K., Rietkerk,

T., Diederen, K. M. J., Bakker, S., Wijkstra, J., and Boks, M. P. M. (2010). Healthy individuals with auditory verbal hallucinations: who are they? psychiatric assessments of a selected sample of 103 subjects. Schizophr. Bull. 36, 633-641.

van der Meer, L., van't Wout, M., and Aleman, A. (2009). Emotion regulation strategies in patients with schizophrenia. Psychiatry Res. 170, 108-113.

Versmissen, D., Janssen, I., Johns, L. McGuire, P., Drukker, M., à Campo, J., Myin-Germeys, I., van Os, J., and Krabbendam, L. (2007). Verbal self-monitoring in psychosis: a non-replication. Psychol. Med. 37, 569-576.

Conflict of Interest Statement: The author declares that the research was conducted in the absence of any commercial or financial relationships that could be construed as a potential conflict of interest.

Received: 21 December 2011; accepted: 07 February 2012; published online: 21 February 2012.
Citation: Larøi F (2012). How do auditory verbal hallucinations in patients differ from those in non-patients? Front. Hum. Neurosci. 6:25. doi: 10.3389/ fnhum.2012.00025

Copyright (c) 2012 Larøi. This is an open-access article distributed under the terms of the Creative Commons Attribution Non Commercial License, which permits non-commercial use, distribution, and reproduction in other forums, provided the original authors and source are credited. 